
F. STAUB ${ }^{1, \infty}$
M. BRAUD ${ }^{1}$
J.E. BALMER ${ }^{1}$
J. NILSEN ${ }^{2}$
S. BAJT ${ }^{2}$

\title{
Simultaneous imaging of the near- and far-field intensity distributions of the Ni-like Sn X-ray laser
}

\author{
${ }^{1}$ Institute of Applied Physics, Sidlerstrasse 5, 3012 Bern, Switzerland \\ ${ }^{2}$ Lawrence Livermore National Laboratory, Livermore, CA 94551, USA
}

Received: 27 October 2003/Revised version: 5 January 2004 Published online: 5 March 2004 • (C) Springer-Verlag 2004

ABSTRACT We report two-dimensional near-field imaging experiments of the 11.9-nm Sn X-ray laser that were performed with a set of novel Mo/Y multilayer mirrors having reflectivities of up to $\sim 40 \%$ at normal and at $45^{\circ}$ incidence. Second-moment analysis of the X-ray laser emission was used to determine values of the X-ray beam propagation factor $M^{2}$ for a range of irradiation parameters. The results reveal a reduction of $M^{2}$ with increasing prepulse intensity. The spatial size of the output is a factor of $\sim 2$ smaller than previously measured for the 14.7-nm Pd X-ray laser, while the distance of the X-ray emission with respect to the target surface remains roughly the same.

PACS 42.55.Vc

\section{Introduction}

Since the first demonstration of substantial X-ray laser gain in neon-like selenium [1], the electron-collisional excitation scheme has proven to be the most successful method on the route towards high output power and saturated gain. Saturated operation of soft-X-ray lasers is important because it assures the maximum stimulated-emission power extraction from a given volume of excited plasma. Clear evidence of saturation has been reported in the last few years by a number of authors for the $4 \mathrm{~d} \rightarrow 4 \mathrm{p}, J=0-1$ transition in nickel-like elements at wavelengths between 14.7 and $7.3 \mathrm{~nm}$ [2-4]. The efficiency with which saturation is achieved in the Ni-like lasers has recently been improved by exploiting the prepulse and multiple-pulse techniques [5-7] and, on the other hand, by reducing the pump pulse duration to the picosecond range, combined with traveling-wave irradiation [8]. Reports on the properties of these lasers have included measurements of the output energy, pulse duration, near- and far-field intensity distributions, and divergence [9]. The recent development of novel Mo/Y multilayer X-ray mirrors [10] has opened up a new wavelength range from 7 to $12 \mathrm{~nm}$ for imaging experiments of soft X-rays not accessible so far.

Fax:+41-31/631-3765, E-mail: felix.staub@iap.unibe.ch
In previous experiments, we have demonstrated saturated gain on the $4 \mathrm{~d} \rightarrow 4 \mathrm{p}, J=0-1$ transition in Ni-like $\mathrm{Pd}, \mathrm{Ag}$, and $\mathrm{Sn}$ at $14.7,13.9$, and $11.9 \mathrm{~nm}$, respectively, with a pump irradiation scheme utilizing a $0.5 \%$ prepulse that irradiated flat-slab targets 5-6 ns before the main pulse. Both the prepulse and the main pulse had a duration of 100 ps. In this work we report a series of simultaneous near- and far-field imaging experiments for the Sn 11.9-nm X-ray laser using four different prepulse schemes: a $0.5 \%$ prepulse at $-6 \mathrm{~ns}$, a $2.8 \%$ prepulse at $-2 \mathrm{~ns}$, a $8 \%$ prepulse at $-1 \mathrm{~ns}$, and a $16 \%$ prepulse at $-0.8 \mathrm{~ns}$.

\section{$2 \quad$ Experimental setup}

The experiments were performed using the 1054-nm Nd : glass laser system at the Institute for Applied Physics (IAP) of the University of Berne. The laser, having a final amplifier of $90-\mathrm{mm}$ diameter, is capable of delivering up to $40 \mathrm{~J}$ at a pulse duration of $100 \mathrm{ps}$ (FWHM). The output beam is focused to give a line focus of $2.5-\mathrm{cm}$ length and approximately $100-\mu \mathrm{m}$ width using a combination of a 500-mm focal length aplanatic doublet and a $-1700-\mathrm{mm}$ focal length cylindrical lens. Targets were $25-\mathrm{mm}$-wide slabs having a diamond-machined surface finish. The amplified spontaneous emission (ASE) X-radiation is emitted from either end of the target along the line focus.

The main diagnostics setup is shown in Fig. 1a. It consists of a concave, multilayer, normal-incidence Mo/Y X-ray mirror that images the output aperture at one end of the Sn 11.9-nm laser onto the $\mathrm{P} 43$ phosphor/CCD camera recording system via a flat normal-incidence Mo/Y mirror. The mirrors are made of up to 60 alternating layer pairs of Mo and $\mathrm{Y}$ yielding reflectivities in excess of $40 \%$. The layers have a period of $6.15 \mathrm{~nm}$ for normal incidence and $8.97 \mathrm{~nm}$ for the $45^{\circ}$ mirror, respectively. A detailed description of the multilayers can be found in [10]. The concave mirror has a radius of curvature of $24 \mathrm{~cm}$ and is positioned so as to image the output plane of the Sn laser onto the phosphor screen with a magnification of 12 . The overall spatial resolution of this diagnostics was measured to be $\sim 2.5 \mu \mathrm{m}$ at the output plane of the Sn laser. This value is consistent with the smallest structures that can be resolved in the near-field images. The targets had a 25 - $\mu \mathrm{m}$-diameter steel wire placed $145 \mu \mathrm{m}$ in front of 

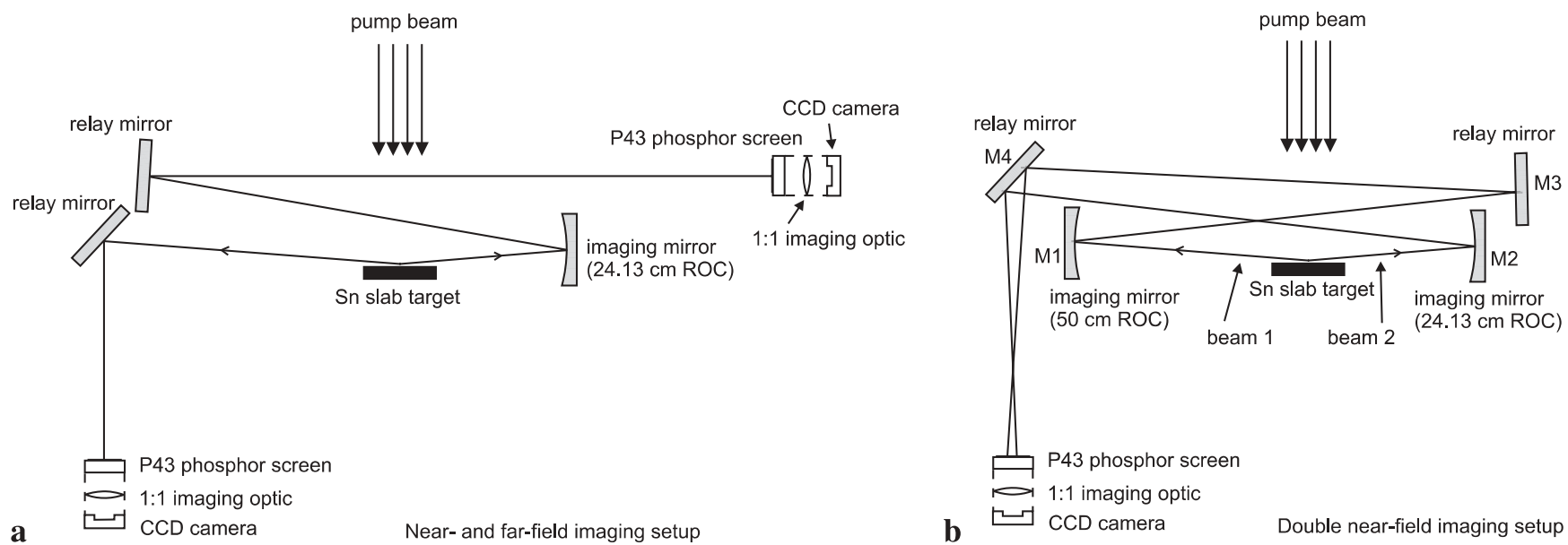

FIGURE 1 a Experimental setup for near- and far-field imaging. The pump beam illuminates the 2.5 -cm-long flat Sn target. The output aperture of the 11.9-nm nickel-like tin laser is imaged with a magnification of 12 onto the P43 phosphor/CCD camera detection system. The beam propagating to the left is used for the far-field diagnostics. b Experimental setup for double-near-field imaging. The pump beam illuminates the 2.5 - $\mathrm{cm}-\mathrm{long}$ flat Sn target. The output apertures of the 11.9-nm nickel-like tin laser are imaged onto the P43 phosphor/CCD camera detection system with magnifications of 10 and 17 , respectively

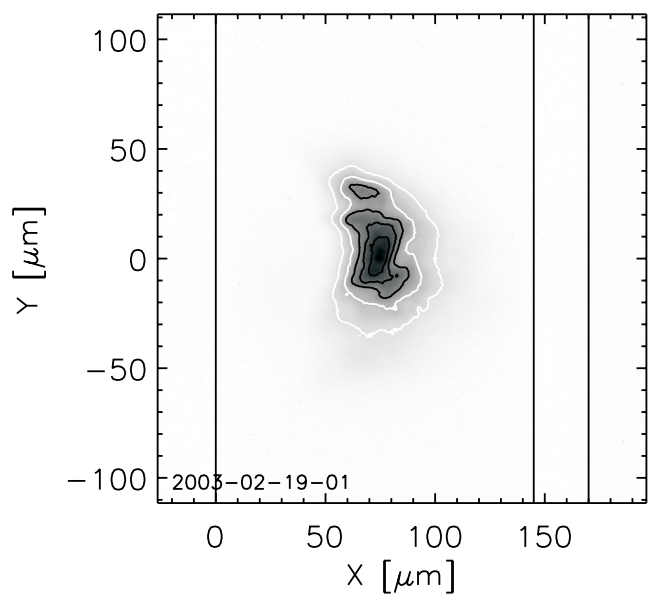

the target surface at an end of the target that served as an absolute spatial fiducial in the measurements. The laser emission from the other end of the target was used to record the far-field intensity distribution at a distance of $2.05 \mathrm{~m}$ from the target via a $45^{\circ}$ angle-of-incidence $\mathrm{Mo} / \mathrm{Y}$ turning mirror (see Fig. 1a). The recording system was a similar P43 phosphor/CCD camera combination. The spatial resolution in this case was given by the $23-\mu \mathrm{m}$-square pixel size of the CCD camera. In both cases, the $\mathrm{P} 43$ phosphor was coated with $\sim 120 \mathrm{~nm}$ of zirconium to discriminate against background radiation.

A second diagnostics setup is shown in Fig. 1b. It consists of two concave, multilayer, normal-incidence $\mathrm{Mo} / \mathrm{Y}$ $\mathrm{X}$-ray mirrors that image the output apertures at both ends of the Sn 11.9-nm laser onto the $\mathrm{P} 43$ phosphor/CCD camera recording system via a flat normal-incidence and a $45^{\circ}$ angleof-incidence $\mathrm{Mo} / \mathrm{Y}$ mirror. The concave mirrors have radii of curvature of $50 \mathrm{~cm}$ and $24 \mathrm{~cm}$, respectively, and are positioned so as to image the output planes of the Sn laser onto the phosphor screen with magnifications of 10 and 17, respectively. The targets had 25- $\mu \mathrm{m}$-diameter steel wires placed $100 \mu \mathrm{m}$ in front of the target surface that served as absolute spatial fiducials in the measurements.
FIGURE 2 Left: near-field image of the Sn laser emission (contour plot). The plasma blow-off direction is along the horizontal axis with zero corresponding to the original target surface. Right: corresponding far-field image (contour plot), showing modulations along the $Y$ axis
3

Figure 2 shows typical images of the Sn $J=0-1$ laser emission at $11.9 \mathrm{~nm}$ at the output aperture of the target (left) and the simultaneously recorded corresponding far field (right) at a drive irradiance of $10 \mathrm{TW} / \mathrm{cm}^{2}$ and a $0.5 \%$ prepulse preceding the main pulse by $6 \mathrm{~ns}$. The plasma blow-off direction is the horizontal axis in the left-hand figure with zero corresponding to the original target surface. The small-scale structures observed in the near-field image are most likely caused by inhomogeneities in the density profile and/or by the different gain lengths experienced by the individual rays. The modulations seen in the far-field images are attributed to coherent rays, spatially overlapping in the gain region, but diverging by 10 to $20 \mu \mathrm{rad}$, forming an interference pattern in the far field. The pattern was reproducible for most of the shots for unchanged prepulse conditions, with the strongest modulations observed with the $0.5 \%$ prepulse. With increasing prepulse amplitude the modulations of the far-field showed a tendency to become weaker and more irregular. The angular frequency and the modulation depth are considerably lower compared to the interference fringes observed in the far-field beam patterns of $[11,12]$. 
A series of measurements was performed for prepulse amplitudes of $0.5,2.8,8$, and $16 \%$. Assuming the X-ray laser emission characteristics to be similar on both ends of the target, a second-moment analysis of the intensity distributions was used to determine the X-ray laser beam propagation factors, $M_{x}^{2}$ and $M_{y}^{2}$, in the directions perpendicular and parallel to the target surface, respectively. The beam propagation factors are given by [13]

$M_{x}^{2} \equiv \frac{\pi \Delta x \Delta \theta}{\lambda}, \quad M_{y}^{2} \equiv \frac{\pi \Delta y \Delta \vartheta}{\lambda}, \quad M^{4} \equiv M_{x}^{2} M_{y}^{2}$,

where $\lambda$ is the laser wavelength, $\Delta x$ and $\Delta y$ are the near-field beam radii in horizontal and vertical directions, respectively, and $\Delta \theta$ and $\Delta \vartheta$ the corresponding divergence semi-angles given by

$\Delta x=2 \sqrt{\left\langle x^{2}\right\rangle-\left\langle x^{1}\right\rangle^{2}}, \quad \Delta \theta=2 \sqrt{\left\langle\theta^{2}\right\rangle-\left\langle\theta^{1}\right\rangle^{2}}$.

The $n$th moments of a beam for the $x$ and $\theta$ coordinates are defined as follows:

$\left\langle x^{n}\right\rangle=\frac{\iint x^{n} I_{S}(x, y) \mathrm{d} x \mathrm{~d} y}{\iint I_{S}(x, y) \mathrm{d} x \mathrm{~d} y}, \quad\left\langle\theta^{n}\right\rangle=\frac{\iint \theta^{n} I_{a}(\theta, \vartheta) \mathrm{d} \theta \mathrm{d} \vartheta}{\iint I_{a}(\theta, \vartheta) \mathrm{d} \theta \mathrm{d} \vartheta}$,

where $I_{s}(x, y)$ and $I_{a}(\theta, \vartheta)$ are the near-field and the far-field intensity distributions, respectively. The calculation for the $y$ and $\vartheta$ coordinates is analogous.

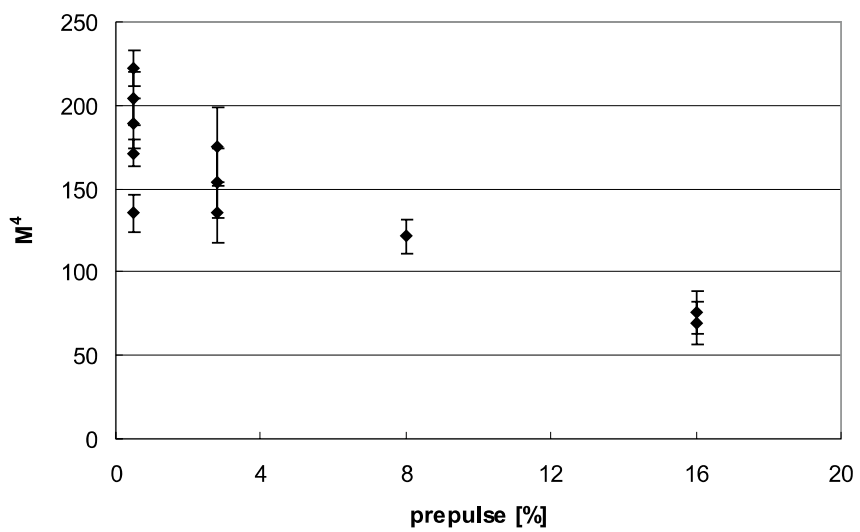

FIGURE 3 Sn X-ray laser beam propagation factor $M^{4}=M_{x}^{2} M_{y}^{2}$ as a function of prepulse amplitude

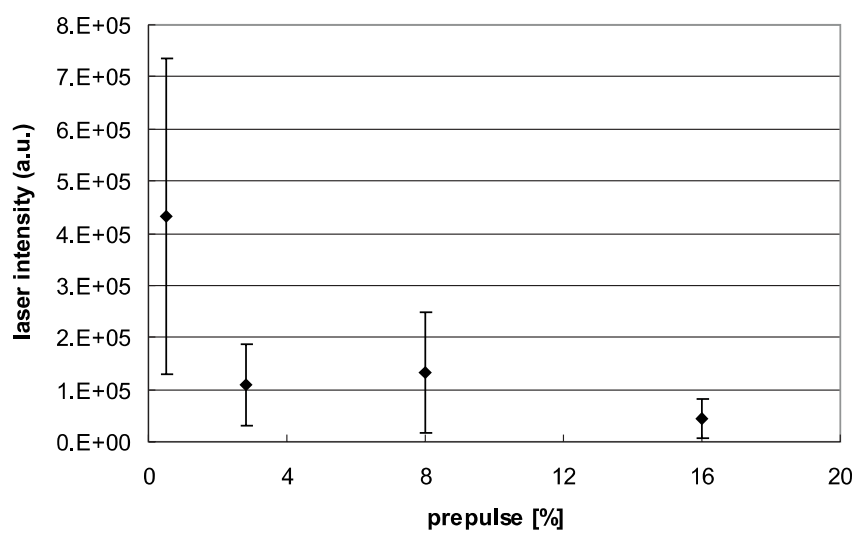

FIGURE 4 Sn X-ray laser intensity as a function of prepulse amplitude

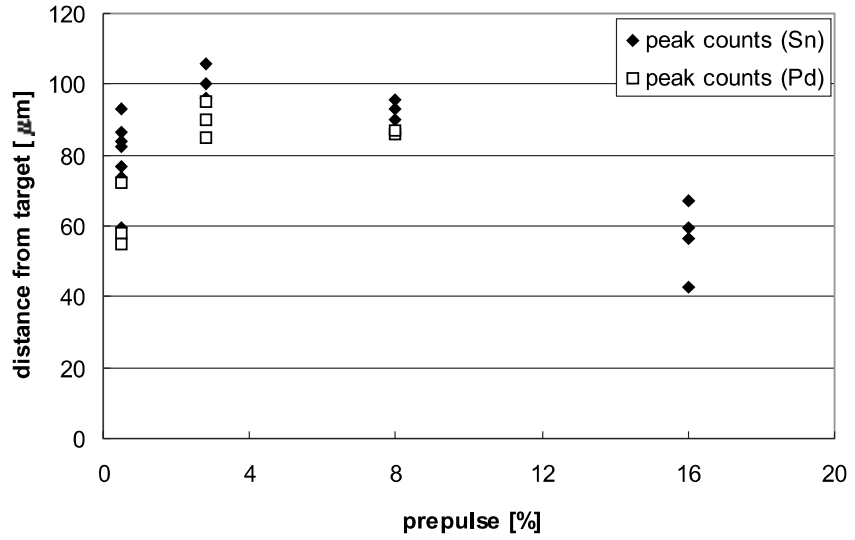

FIGURE 5 Distance of the 11.9-nm Sn X-ray laser emission zone from the target as a function of prepulse amplitude. Results for the 14.7-nm Pd X-ray laser from [14] are included for comparison

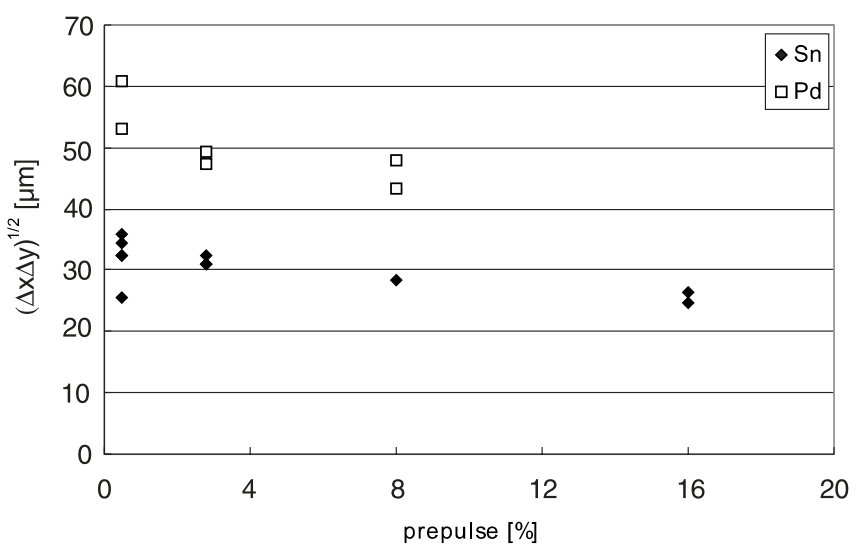

FIGURE 6 Mean radius of the near-field intensity distribution based on second-moment analysis as a function of prepulse amplitude. Results for the Pd X-ray laser are included for comparison

The results are plotted in the form of the product $M^{4}=$ $M_{x}^{2} M_{y}^{2}$ in Fig. 3. As can be seen, the value of $M^{4}$ decreases with increasing prepulse amplitude, accompanied by a decrease in the X-ray intensity (Fig. 4). More detailed analysis shows that the decrease of $M^{4}$ is due to a reduction in both the size of the emitting zone and the divergence of the laser beam. This is consistent with the findings of [14] for the 14.7-nm Pd $\mathrm{X}$-ray laser.

With the aid of the fiducial wire, the position of the emitting zone was measured for the four prepulse configurations investigated. Figure 5 shows a plot of the distance of the emission zone from the original target surface as a function of the prepulse amplitude, together with the results of the Ni-like Pd X-ray laser presented in [14]. As can be seen, the curve shows a flat maximum at $\sim 100 \mu \mathrm{m}$ from the target for a prepulse amplitude of $\sim 3 \%$. Figure 6 shows a plot of the geometrical mean radius of the emission zone as a function of the prepulse amplitude. The emission zone for the Pd laser is about a factor of 1.5 to 2 larger than for $\mathrm{Sn}$.

In order to verify that the laser emission characteristics are similar on both ends of the target, the near fields at both ends were recorded simultaneously (setup as in Fig. 1b). We found that for most of the shots both near fields are similar in size (correlation coefficient $R=0.35)$ and orientation $(R=0.42)$, 
while a few rather asymmetric images exhibit a complementary intensity distribution. The peak intensities of both near fields are well correlated $(R=0.96)$. On average, one beam was emitted $\sim 10 \mu \mathrm{m}$ closer to the target surface than the other one. However, the absolute position of the emission has no influence on the moments and does not change the values of the beam propagation factor $M^{2}$.

\section{4}

\section{Conclusions}

We have reported a series of simultaneous nearand far-field imaging experiments for the nickel-like 11.9-nm Sn X-ray laser using novel Mo/Y multilayer mirrors. The measurements were conducted with the aim to characterize the two-dimensional source intensity distribution and its position relative to the target surface. The images were analyzed in terms of the beam propagation factor, $M^{2}$, and the effect of different prepulse amplitudes was investigated. The distance of the emission zone from the target was found to be of the order of $60-100 \mu \mathrm{m}$, with the largest values reached for a few-percent prepulse amplitude. Simultaneous imaging of the near fields yielded evident correlation of the beam parameters for both emitting apertures.

ACKNOWLEDGEMENTS The authors would like to thank H.P. Weber for fruitful discussions, B. Locher for expert technical assistance, and W. Lüscher for target preparation. This work was supported in part by the Swiss National Science Foundation. The work of the LLNL authors was performed under the auspices of the U.S. Department of Energy by the University of California Lawrence Livermore National Laboratory under Contract No. W-7405-Eng-48.

\section{REFERENCES}

1 D.L. Matthews, P.L. Hagelstein, M.D. Rosen, M.J. Eckart, N.M. Ceglio, A.U. Hazi, H. Medecki, B.J. MacGowan, J.E. Trebes, B.L. Whitten, E.M. Campbell, C.W. Hatcher, A.M. Hawryluk, R.L. Kauffman, L.D. Pleasance, G. Rambach, J.H. Scofield, G. Stone, T.A. Weaver: Phys. Rev. Lett. 54, 110 (1985)

2 J. Zhang, A.G. MacPhee, J. Nilsen, J. Lin, T.W. Barbee, Jr., C. Danson, M.H. Key, C.L.S. Lewis, D. Neely, R. M. N. O'Rourke, G.J. Pert, R. Smith, G.J. Tallents, J.S. Wark, E. Wolfrum: Phys. Rev. Lett. 78, 3856 (1997)

3 J. Zhang, A.G. MacPhee, J. Lin, E. Wolfrum, R. Smith, C. Danson, M.H. Key, C.L.S. Lewis, D. Neely, J. Nilsen, G.J. Pert, G.J. Tallents, J.S. Wark: Science 276, 1097 (1997)

4 R. Tommasini, F. Loewenthal, J.E. Balmer: Phys. Rev. A 59, 1577 (1999)

5 J. Nilsen, B.J. MacGowan, L.B. Da Silva, J.C. Moreno: Phys. Rev. A 48, 4682 (1993)

6 H. Daido, Y. Kato, K. Murai, S. Ninomiya, R. Kodama, G. Yuan, Y. Oshikane, M. Takagi, H. Takabe, F. Koike: Phys. Rev. Lett. 75, 1074 (1995)

7 J.E. Balmer, R. Tommasini, F. Loewenthal: IEEE J. Quantum Electron. QE-5, 1435 (1999)

8 J. Dunn, Y. Li, A.L. Osterheld, J. Nilsen, J.R. Hunter, V.N. Shlyaptsev: Phys. Rev. Lett. 84, 4834 (2000)

9 J. Nilsen, J. Zhang, A.G. MacPhee: Phys. Rev. A 56, 3161 (1997)

10 J. Nilsen, S. Bajt, H.N. Chapman, F. Staub, J.E. Balmer: Opt. Lett. 28, 2249 (2003)

11 J. Dunn, R.F. Smith, J. Nilsen, J.R. Hunter, T.W. Barbee, Jr., V.N. Shlyaptsev, J. Filevich, J.J. Rocca, M.C. Marconi, H. Fiedorowicz, A. Bartnik: Proc. SPIE 4505, 62 (2001)

12 A. Klisnick, J. Kuba, D. Ros, A. Carillon, G. Jamelot, R. Smith, F. Strati, G.J. Tallents, R. Keenan, S.J. Topping, C.L.S. Lewis, P. Nickles, K.A. Janulewicz, F. Bortolotto, D. Neely, R. Clarke, J. Collier, A.G. MacPhee, C. Chenais-Popovics, J.-C. Chanteloup, D. Joyeux, D. Phalippou, H. Daido, H. Tang: Proc. SPIE 4505, 75 (2001)

13 A.E. Siegman: Proc. SPIE 1224, 2 (1990)

14 C. Siegel, M. Braud, J.E. Balmer: Opt. Commun. 210, 305 (2002) 